\title{
Low Dose Defocused Probe Electron Ptychography Using a Fast Direct Electron Detector
}

\author{
Christopher S. Allen ${ }^{1,2}$, Jiamei Song ${ }^{3}$, Mohsen Danaie ${ }^{2}$, Peng Wang ${ }^{3}$ and Angus I. Kirkland ${ }^{1,2}$ \\ 1. Department of Materials, University of Oxford, Parks Road, Oxford, UK. \\ 2. Electron Physical Sciences Imaging Centre (ePSIC), Diamond Light Source, Didcot, UK. \\ 3. National Laboratory of Solid State Microstructures, College of Engineering and Applied Sciences and \\ Collaborative Innovation Center of Advanced Microstructures, Nanjing University, Nanjing, P.R.C.
}

The advent of fast direct-electron detectors has expanded the available dataset of a STEM experiment from an integer to a two-dimensional diffraction pattern at each probe position in a two dimensional raster scan. One way to exploit these four dimensional data-sets is to ptychographically retrieve the complex specimen exit surface wave function [1]. This exit wave function contains phase information which is not directly accessible using conventional STEM imaging techniques. The ability to quantitatively recover the phase of the exit wave enables the simultaneous imaging of both light and heavy atoms [2,3] as well as accessing three dimensional information normally absent from projection STEM imaging $[2,4]$.

Electron ptychography has been demonstrated using a focused STEM probe [5]. The development of fast direct-electron detectors combined with the stability of modern electron microscopes has enabled the ptychographic reconstruction from several hundreds of thousands of probe positions. However, even with fast cameras the dwell time per probe position is still of the order of hundreds of $\mu \mathrm{s}$, orders of magnitude longer than for conventional ADF imaging. As a result the cumulative dose on the sample is typically high and the reconstructions are susceptible to drift in both the sample and lenses. A further challenge is presented by the very large data-sets collected. To combine satisfactory sampling with reasonable field of view probe arrays equivalent to those used in conventional ADF imaging are required and these data-sets quickly run in to several tens or even hundreds of giga-bytes per image.

An alternate technique is to scan a probe defocused by several tens of nm's over the sample surface with highly overlapping probe positions. Using this technique just a few tens of probe positions are required to successfully reconstruct the phase. Defocused probe ptychography has shown success in both imaging light $\mathrm{B}$ atoms in $\mathrm{a}^{\mathrm{LaB}_{6}}$ crystal [2] and retrieving three dimensional structural information of a nanostructured material [3]. This defocused probe approach has the disadvantage that it is not compatible with standard STEM ADF or spectroscopic techniques. However, the exemplary detection characteristics and high speed of direct electron detectors combined with the defocused nature of the probe and few probe positions offer the potential of atomic resolution quantitative phase imaging of beam sensitive samples as well as greatly limiting the size of the collected data set.

Here we use a 256x256 array Merlin-Medipix direct electron detector to collect diffraction patterns from a defocused electron probe incident on a monolayer $\mathrm{MoS}_{2}$ sample. By ptychographically reconstructing a phase image we demonstrate a resolution enhancement at low dose compared to standard ADF STEM imaging.

Figure 1(a) shows a focused-probe ADF of monolayer $\mathrm{MoS}_{2}$ recorded at $80 \mathrm{keV}$ at a beam current of $1 \mathrm{pA}$, convergence semi angle of $24 \mathrm{mrad}$ and inner collection semi-angle of $46 \mathrm{mrad}$. This image was recorded with an electron dose of $5 \times 10^{3} \mathrm{e}^{-} / \mathrm{A}^{2}$. The relatively heavy Mo atoms are resolved but the contrast of 
the $\mathrm{S}_{2}$ positions falls below the Rose criterion. In the two-dimensional power spectrum the (200) reflections are visible demonstrating a resolution of $136 \mathrm{pm}$ (Figure 1c.). For weak scattering, low dimensional materials such as $\mathrm{MoS}_{2}$ this is close to the limit of low dose imaging for standard ADF STEM. This is however still one to two orders of magnitude higher than that typically used in the imaging of biological samples.

By moving the focal point of the probe to approximately $80 \mathrm{~nm}$ below the plane of the sample a probe of approximately $4 \mathrm{~nm}$ diameter was formed. The defocused probe was raster scanned across the sample in an array of $10 \times 10$ probe positions with step size of approximately $0.96 \mathrm{~nm}$. The beam current was $17 \mathrm{pA}$ and dwell time at each probe position $4 \mathrm{~ms}$. This illumination corresponded to a electron dose of $2 \times 10^{3}$ $\mathrm{e}^{-} / \mathrm{A}^{2}$, half of that used to record the ADF image shown in Figure 1a. Using the ePIE algorithm [6] the phase of the exit wave function was ptychographically reconstructed. The phase image is shown in Figure 1b. Both the Mo and the $\mathrm{S}_{2}$ atomic positions are clearly resolved with the (200) reflection (136 pm) visible in the two dimensional power spectrum (Figure 1d.).

This study demonstrates the potential for defocused probe ptychography for low dose imaging of beam sensitive materials [7].

\section{References:}

[1] JM Rodenburg, In “Advances in Imaging and Electron Physics vol. 150”, ed. Hawkes, (Elsevier) p. 87.

[2] H Yang et al, Nature Communications 7 (2016), p. 12532.

[3] P Wang et al, Scientific Reports 7 (2017), p. 2857.

[4] S Gao et al, Nature Communications 8 (2017), p. 163.

[5] H. Yang, H. et al, Ultramicroscopy 180 (2017), p. 173.

[6] AM Maiden and JM Rodenburg. Ultramicroscopy 109 (2009), p. 1256

[7] We thank Diamond Light Source for access and support in use of the electron Physical Science Imaging Centre (EM17918) that contributed to the results presented here.
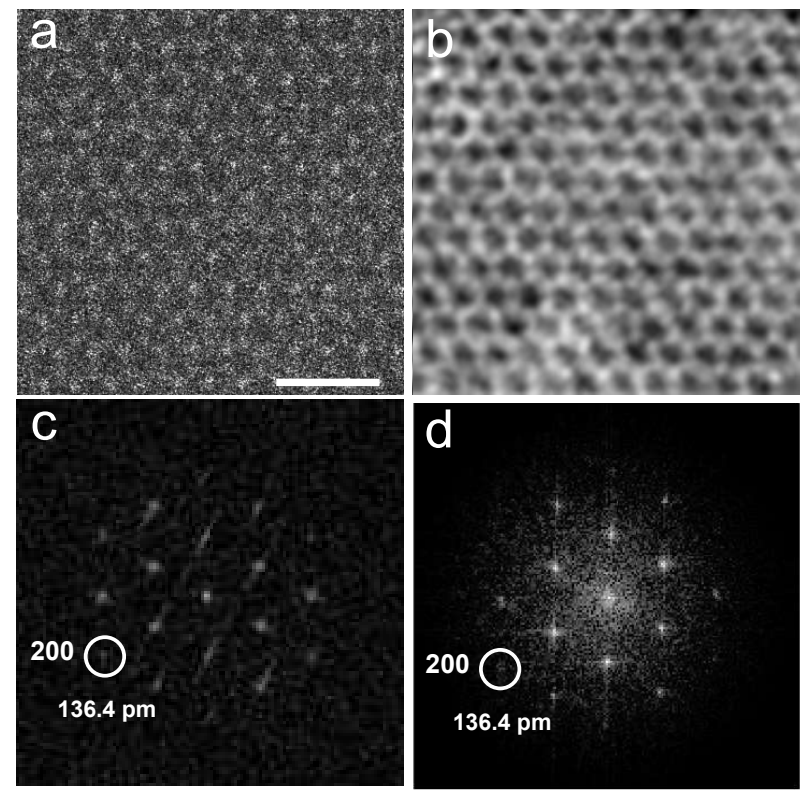

Figure 1. (a) HAADF image of a mono-layer $\mathrm{MoS}_{2}$ with a dose of $4.9 \times 10^{3} \mathrm{e}^{-} / \mathrm{A}^{2}$; (b) Phases of the ptychographic reconstructions from the same region with a dose of $5.0 \times 10^{3} \mathrm{e}^{-} / \mathrm{A}^{2}$; (c-d) power spectra of (a-b) respectively displayed on a logarithmic intensity scale. 